Int Arch Allergy Immunol 1997;112:427-428

\title{
Subject Index, Vol. 112, 1997
}

Adenosine 83 Adhesion molecule 287 Afferent neurons 303 Affinity-purified antiphospholipid antibodies 19 Ag(e)ing 196, 323 Airflow obstruction 196 Airway inflammation 191,295,386,406 - $\quad$ sensitivity 59

Allergen(s) 44, 88, 133, 169, 341, 371, 378 Allergen-specific conjunctival challenge

247 Allergic aspergillosis 270

asthma 247

inflammation 247

rhinitis 400 Allergy 65,331,341 Alternative medicine 238 Amyloidosis 415 Anti-endomysial antibody 140 Antigen processing 103 Antigen-presenting cells 103,317 Antiphospholipid syndrome 19 Apoptosis 9

Aspergillus antigen 270

- umbrosus 313

Asthma 65, 196, 295, 303, 309, 406 Atopic dermatitis 163, 238 Atopy 49 Autoimmune disease 422

B cells 49

Baker's asthma 378

Basophils, human 336

Biological properties 218

Bioresonance therapy 238

Birch pollen-specific IgE 175

Blomia tropicalis 341

Bosd2 231

Bromelain 412

Bronchial asthma 59,287,336

C fibers 303

epithelial cells 157

provocation test 386

responsiveness 191 Bronchoalveolar lavage 295, 309

C5adesarg 27 Candida albicans 313 Carbohydrate moiety 412 CD4+T cells 125 CD40L 49

CD80 expression defect 96

Cell biology 103

injury 262

lysate histamine 309 Chit 1-9 133 cICAM-1 386

c-kit receptor 36

Clinical course 163

Clustering 371

Coeliac disease 140

Common variable immunodeficiency 96

Contact hypersensitivity 257 
Cow hair asthma 231

Cross-reactivity 169, 313, 371, 378, 412

Cyclosporin(e) A 279, 422

Cytokine(s) 9,270, 323, 392

- $\quad$ gene expression 73

Degranulation 392 Demethoxyviridin 392 Dendritic cells 317 Derfl 365 Derf2 44

Dermatophagoidesfarinae 73 Dexamethasone 13

Early-phase reaction 247

Edema 196

EG2 59

En dothelial cells 145

Endothelin 83

Enzymatic activity 226

Eosinophil(s) 9, 73, 270, 287, 295, 309, 406

- $\quad$ cationic protein 59, 386

Epidermal cells 125

Erythromycin 262

Exocytosis 336

Extrinsic allergic alveolitis 313

Farmer's lung disease 313 Fatty acid binding protein 341 FK506 422

Flow cytometry 125, 133 fMLP 27

G protein 336 Gene induction 392 Genomic sequence 44 Glucocorticoids 279 Grass allergy 184 - $\quad$ pollen 378

Guinea pig 406

Half-life 175

Hematopoietic progenitor cell 36

stem cells 3 Hen's egg allergy 163 Histamine 88, 145, 196 HLA 365 HLA-B8, DR3 93 House

dust 231

- mite 44

Human endothelial cell 262

- neutrophil 262

Hymenoptera venom anaphylaxis 251

IgE 36, 49, 88, 163, 226, 279, 313, 331, 348,422

- $\quad$ myeloma 415

- , total 175

IgG 279

Immunoblotting 169 Immunoglobulin 331

- $\quad$ E 341

Immunosuppression 257 Immunotherapy 184 Inflammation 9, 115 Influenza 323 Inhibition 378 Intercellular adhesion molecules

145 Interferon- $\gamma$ 152,323 Interleukin-4 49, 152, 323, 422 Interleukin-5 73 Interleukin-8 157

Interleukin-10 13, 152 Interleukin-13 49 Intraembryonic origin 3 Intranasal pressure 400

Isoallergen 348

Kawasaki disease 152

Langerhans cells 125 Late asthmatic response 287 Leukocytes 115 Leukotriene(s) 392

- $\quad$ B4 27 
Lolium perenne 184 Lolpl 218,412 Lymphocyte(s), human 212

proliferative response 163

response 133

Macrophages 27

Major histocompatibility class II 145

KAHGEII

(C) 1997 S. KargerAG, Basel

427

E-Mail karger@karger.ch Fax+4161306 1234 http://www.karger.ch

Mast cell(s) 27, 392

- $\quad$ - progenitor 36

Measures of avoidance 231

Melatonin effects 203

-, extrapineal synthesis 203 -, immune system 203 -, lymphatic system 203 -, mechanisms of action 203 -, physiological role 203

- $\quad$ receptors, immune system 203

Membrane metalloendopeptidase 303

Mercurialis annua 356

Metals 212 Mice 73, 125 Mites 295

Monodelphis domestica 257 Mononuclear cells 13 Morphea 93 Mosquitoes 169 Mucosal immunity 103 Mycobacterium tuberculosis, purified protein derivative 323

Nasal allergy 36

blockage 400

mucosa 36 Natural killer 331

- cell activity 331 Neutropenia, cyclic 96 Neutrophil(s) 406

elastase 157,262

migration 27

Nonspecific bronchial responsiveness 65

Ovalbumin 196 Ozone 191

Paraproteinuria 415 Parietaria judaica 348

- $\quad$ - pollen 247

Patch-clamp technique 336

Peptide(s) 226, 365

Peripheral blood lymphocytes, human 175

- $\quad$ - mononuclear cells, antibody

responses 184 Phagocytes 115

Phosphatidylinositol-3-kinase 392 Phosphatidylserine, age-degraded 19 Phosphodiesterase inhibitor 406 Phospholipase(s) 115

- $\quad$ A2 226

Photoreactivating light 257 Pineal immune functions 203 Plasma exudation 196 Platinum compounds 125 Pollen 88,218,371

allergy 356

antigenic cross-reactivity 356 Pollinosis 378 Polymorphisms 44

Popliteal lymph node assay 125 Profilin 356

Pseudomonas aeruginosa 115 Pulmonary function 59 
- $\quad$ inflammation 73

Radioimmunoassay 251 Receptor-mediated endocytosis 125 Recombinant allergen 218,341,348

Relevant allergen 356 Renal insufficiency 415 Renin-angiotensin system 251 Rhinitis 65 RNA

13

S-1452 400

Saliva proteins 169

E-Selectin 145

Sensitisation 279

Serotonin 145

Severe combined immunodeficiency 175

Signal transduction 9

Silicone implant 93 Skintest(s) 212,371 Specific IgE 378 Specificity 19 Sputum, induced 386

Sting provocation 251

Tcell(s) 49,88

- epitope(s) 226,365

- receptor 133

cell-mediated immunity 317

lymphocytes 323 Tetanus toxoid 323 Thl 152

Th2 152

- $\quad$ cells 270

Theophylline 83

Thromboxane A2 400

TNF $\alpha 392$

Tolerance 317

Total IgE 331

Travelling salesman problem 371 Trimellitic anhydride 279 Tryptase 36 Tyrosine kinases 9

Umbilical cord, human 140 Urinary trypsin inhibitor 157 Urocanicacid 257 UVA radiation 257

Vaccination 317

Vagus nerve 303

Vascular permeability 196

Vasculitis 96

Very late activation antigen-4 287

Wheat flour 378 Wortmannin 392

428

Int Arch Allergy Immunol 112, 1997

Subject Index 